\title{
Pricing Options Using Trinomial Lattice Method
}

\author{
Kenneth Kiprotich Langat ${ }^{1, *}$, Joseph Ivivi Mwaniki ${ }^{1}$, George Korir Kiprop ${ }^{2}$ \\ ${ }^{1}$ School of Mathematics, University of Nairobi, Kenya \\ ${ }^{2}$ Pan African University, Institute of Basic Sciences, Technology and Innovation, Nairobi, Kenya \\ *Corresponding author: langatken26@gmail.com
}

Received May 10, 2019; Revised June 18, 2019; Accepted July 02, 2019

\begin{abstract}
How much to spend on an option contract is the main problem at the task of pricing options. This become more complex when it comes to projecting the future possible price of the option. This is attainable if one knows the probabilities of prices either increasing, decreasing or remaining the same. Every investor wishes to make profit on whatever amount they put in the stock exchange and thus the need for a good formula that give a very good approximations to the market prices. This paper aims at introducing the concept of pricing options by using numerical methods. In particular, we focus on the pricing of a European put option which lead us to having American put option curve using Trinomial lattice model. In Trinomial method, the concept of a random walk is used in the simulation of the path followed by the underlying stock price. The explicit price of the European put option is known. Therefore at the end of the paper, the numerical prices obtained by the Black Scholes equation will be compared to the numerical prices obtained using Trinomial and Binomial methods.
\end{abstract}

Keywords: options, random walk, trinomial lattice model, Black-Scholes.

Cite This Article: Kenneth Kiprotich Langat, Joseph Ivivi Mwaniki, and George Korir Kiprop, "Pricing Options Using Trinomial Lattice Method." Journal of Finance and Economics, vol. 7, no. 3 (2019): 81-87. doi: $10.12691 /$ je- $7-3-1$.

\section{Introduction}

\subsection{Background of the Study}

The idea of an option contract can be drawn back to before 1973. These contracts were seen as Over-thecounter (OTC) [1]. This means that option trading had an intermediary, which is the option broker. This option broker is the person who negotiates the price of the option between the buyer and the seller every time an option was to be bought. These option contracts were not handled properly since the contracts were not standardized in expressions of its conditions. The OTC could manage to handle it because few companies were involved. Later in 1973 official exchange begun when the modern financial option market came into the market and quickly replaced the OTC. In the same 1973, the Black-Scholes pricing model was formulated by Fisher Black and Myron Scholes. An option is a contract between two parties that gives the buyer (owner) the right but not the obligation to buy or sell the underlying asset for the agreed cost (strike price) on or before expiring time of the contract. The main types of contracts are call option and the put option. Call option refer to buying of stock whereas put refer to selling the underlying stocks. America option allows the holder chances to exercise it before the agreed deadline. This early exercise leads to a free boundary problem. On the other hand, European option restrict the holder from exercising its rights until the expiring date. Pricing options has been a major field of research in financial mathematics for many years. The main method of pricing was brought up by Black and Scholes and was used for many years before the other methods like numerical methods which include finite difference, Montel Carlos, Binomial, Trinomial and many other methods. Trinomial model has been used in few options like American option, European option and also in exotic options like lookback, barrier and Asian option. Among these exotic options, we have the Russian option which is rarely in the stock exchange market. Trinomial tree model is just an extension of Binomial model from having two branches of either price of a share going up or decreasing to having three branches of either price of the stock increases, remaining the same or decreases. When a trinomial tree model is recombined, we get a trinomial lattice. This means that all nodes that end up with the same prices at the same time will be taken to be one node. In this lattice every node has three possible paths to follow, going up, going down or remaining same. This is out of the results of multiplying the stock price at the node by either of the three factors $u, d$ and $m$. These are ratios whereby $u$ is greater than one, $d$ is less than one and $m$ is equal to one. Apart from these ratios, we have the risk-neutral probabilities which tell us the chance that a price has to increase, decrease or remain the same. The price of an option is derived using this trinomial lattice by starting from the last price or the expiration time price by discounting one step backward. The same process is repeated all the way till the price at time zero is gotten and that is the price of the options. 


\subsection{Statement of the Problem}

For any individual or organization that make any investment in any business, the main focus is on making profit at the end of the day. The same case applies to investors who put their money into stock exchange with the intention of adding value to their money invested there in future, an example is trading in option contracts. Business is a risk that can either end up making profit or loss. Every investor wish to make profit and so to increase the chance of this, the investor should be in a good position of making the right decisions. Prior to making the decision of buying an option in the stock exchange, the investor should be able to know the price that he/she should pay today. The investor then asked himself, "How much should I pay for an option?" This is a factor considered on selection of shares in the market by the factor of optimal prices. An investor will seek to get a stock that is very marketable and with less risk of price dropping from the current price. This will largely lead to profit making and thus the need of right judgement of the current price.

\section{Literature Review}

In the past many year, option pricing has been the main area of research in the financial studies. The famous pricing option model being the Black and Scholes found in [2]. This model has a lot of mathematics which requires a lot of knowledge in mathematics and thus not friendly to many people who doesn't have good background of mathematics. Later on, [3] came up with article "Theory of rational option pricing" which gave them a breakthrough in the pricing of option and thus coming up with the "Black-Scholes-Marton" formula. Thereafter, [4] introduce the binomial pricing option model which is easy to understand due to its simple mathematics and its implicit economic importance and thus majorly used in the financial markets. On the other hand, this model could lead to large errors because it only allow two ways states, that is stock price of underlying asset move up or move down from one step to another. To help in doing away with such errors, [5] came up with the Trinomial pricing model. This was modified by Boyle and Lau in [6] and showed their solutions. They suggest that there would be three varying prices of the underlying asset at a certain time step which are either price moving up, remaining the same or moving down. Here they prove that trinomial tree model is more realistic than binomial model. This makes trinomial model better option for pricing options since it is more accurate in solution and at the same time converge faster than binomial hence making trinomial tree model widely used in pricing different types of options. Han worked on a trinomial tree model for pricing options on specific cases in numerical methods and did comparison with the binomial model and got that trinomial model is better than binomial model in terms of approximating the continuous distribution of the underlying asset price movements with more states and higher accuracy [7]. AitSahlia and Lai in [8] took assumption that the interest rates follows a Markov chain process and came up with a different pricing option formula. Under this formula, they did comparison of trinomial tree model and binomial model in terms of rate of convergence based on number of nodes produced and the approximation error. They use an example to show that trinomial tree model was more accurate than binomial tree model. Bath and Kumar in the article [9] raised a Markov tree model for pricing option using a non-iid process which is a modification of standard binomial pricing model that take into account first order Markov behavior. Next was pricing option under a normal mixture distribution which is from Markov tree model and concluded that the mixture of two normal distribution is much better than a single normal distribution in terms of fitness. XIONG Bing-zhong in the article [10] came up with a trinomial tree model for pricing options which was based on Bayesian Markov chain Monte Carlo method and used it for comparison of the Black-Scholes model, classic binomial tree model, classic trinomial tree model and the warrant price using the real data from warrant market. The outcome proof that although all methods underestimate the market price, the price difference of trinomial tree model was very small than any other. Young and Yuen in the article [11] proposed tree model to price simple and exotic options in Markov regime switching model (MRSM) with multiregime. They modified Boyle's trinomial tree model by controlling the risk-neutral probabilities measure at different regime states in order to ensure that the tree structure is maintain and at the same time for the model to accommodate the data of all regimes.

\section{The Model}

Trinomial tree method is more like binomial tree with slight difference. From each node the price of the underlying stock (share) branch into three new prices, that is, either being more than the previous price, being less than the previous price or remaining the same as the previous price. This majorly depend on the ratios we use to multiply our current price and at the same time the probabilities that we consider.

\subsection{Assumptions}

(i) Time steps are all equal.

(ii) The interest rate used is the risk free rates.

(iii) Probabilities remain the same throughout.

We use call options to demonstrate trinomial Markov tree model. Let $S_{n}$ to be the stock price at time step $n$ and $S_{0}$ to be the stock price at the start of the contract. Let $K$ to be the strike price. When, $n=0$, the stock price will move in three ways i.e increase, remain unchanged or decrease with the following probabilities

$$
\begin{aligned}
& P\left(S_{1}=u S_{0}\right)=P_{1} \\
& P\left(S_{1}=m S_{0}\right)=P_{2} \\
& P\left(S_{1}=d S_{0}\right)=P_{3}
\end{aligned}
$$

When, $n \geq 1$, the stock price move from one step to another in either of the three ways;

$$
S_{n}^{u}=\left(S_{n}>S_{n-1}\right)
$$




$$
\begin{aligned}
& S_{n}^{m}=\left(S_{n}=S_{n-1}\right) \\
& S_{n}^{d}=\left(S_{n}<S_{n-1}\right)
\end{aligned}
$$

Where $S_{n}^{u}$ is the event when the stock price increases from step $n-1$ to step $n, S_{n}^{m}$ is the event that the price of the stock remain the same and $S_{n}^{d}$ is the event that the price of the stock decreases from step $n-1$ to step $n$.

For $n \geq 1$ the conditional probabilities for the stock price to increase, remain same or decrease given the previous prices to have increase, remain same or decrease respectively are given as follow;

$$
\begin{gathered}
P\left(S_{n+1}=u S_{n} \mid S_{n}^{u}\right)=P_{1 u} \\
P\left(S_{n+1}=m S_{n} \mid S_{n}^{u}\right)=P_{2 u} \\
P\left(S_{n+1}=d S_{n} \mid S_{n}^{u}\right)=P_{3 u} \\
P\left(S_{n+1}=u S_{n} \mid S_{n}^{m}\right)=P_{1 m} \\
P\left(S_{n+1}=m S_{n} \mid S_{n}^{m}\right)=P_{2 m} \\
P\left(S_{n+1}=d S_{n} \mid S_{n}^{m}\right)=P_{3 m} \\
P\left(S_{n+1}=u S_{n} \mid S_{n}^{d}\right)=P_{1 d} \\
P\left(S_{n+1}=m S_{n} \mid S_{n}^{d}\right)=P_{2 d} \\
P\left(S_{n+1}=d S_{n} \mid S_{n}^{d}\right)=P_{3 d} .
\end{gathered}
$$

The three factors $u, m$ and $d$ represent different ratios by which the stock price at one node could change to three prices of the three nodes at the next time step. That is, the next stock price at time step $n+1$ would be $u S_{n}$ with the probability of $P_{1 u}$, if the price don't change, it is $m S_{n}$ and the probability is $P_{2 u}$ and finally if the price drop we have $d S_{n}$ with its probability being $P_{3 u}$.

The diagram below shows the Trinomial tree diagram with the possible path of the stock prices in a two time steps.

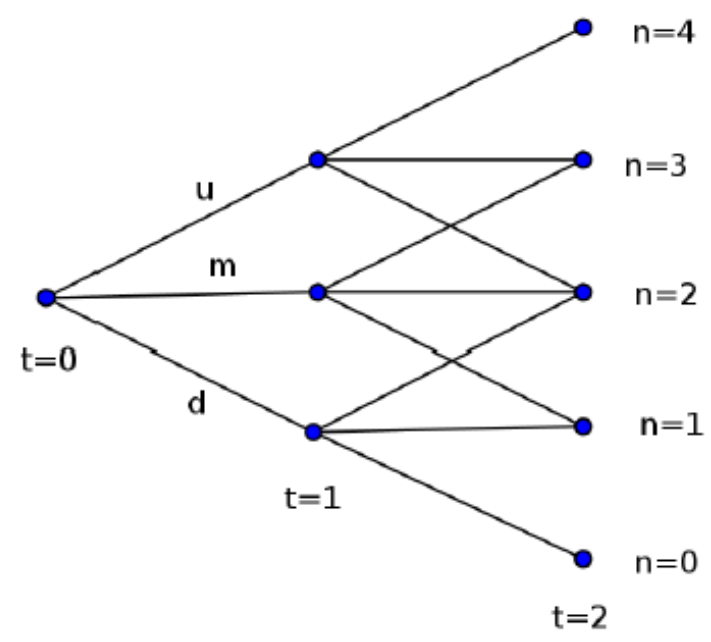

Figure 1. Tree diagram
We know that after a time period, the stock price can move up to $S_{u}$ with probability $P_{u}$, move down to $S_{d}$ with probability $P_{d}$ and (or) remain the same $S_{m}$ with probability $P_{m}$. Thus the corresponding values of call option at the first movement is given by;

$$
\begin{gathered}
f_{u}=\max \left(S_{u}-K, 0\right) \\
f_{m}=\max \left(S_{m}-K, 0\right) \\
f_{d}=\max \left(S_{d}-K, 0\right) .
\end{gathered}
$$

We need to derive a formula that give us a fair price of the option. The way to calculate trinomial tree is just similar to that of binomial tree. You start with the last node and then come to the second last by discounting it. From there, you again discount to reach the third last node. The process continues until you reach the first note. At every node, it is important to calculate the value of holding the option and at the same time knowing the strike price. The value of holding the option is

$$
e^{-r t}\left(P_{u} f_{u}+P_{m} f_{m}+P_{d} f_{d}\right)
$$

while the value of a node is acquired through these three events; $S_{n}^{u}, S_{n}^{m}$ and $S_{n}^{d}$. Since the stock price follows the first-order Markov process, then we will have three types of risk-neutral probability.

Every node end up with three new stock prices after every single time step and thus there are three option prices in which can be denote as; $f_{i, j}^{u}, f_{i, j}^{m}$ and $f_{i, j}^{d}$, where $i=0,1,2, \cdots, n$ is the time steps and $j=-n, \cdots,-1,0,1,2, \cdots, n$ is the vertical position of the nodes.

At time step zero, that is when $i=0$ the option price will be

$$
f=e^{-r \Delta t}\left(P_{u} f_{u}+P_{m} f_{m}+P_{d} f_{d}\right) .
$$

We then extend this model to a two period. We let $f_{u u}$ denote the call value at $2 \Delta t$ for two consecutive upward stock movement, $f_{u d}$ for one upward and one downward, $f_{m m}$ for two consecutive stock prices remaining the same and $f_{d d}$ for two consecutive downward movements.

$$
\begin{gathered}
f_{u u}=\max \left(S_{u u}-K, 0\right) \\
f_{u d}=\max \left(S_{u d}-K, 0\right) \\
f_{m m}=\max \left(S_{m m}-K, 0\right) \\
f_{d d}=\max \left(S_{d d}-K, 0\right)
\end{gathered}
$$

Then the values of the call option becomes;

$$
\begin{gathered}
f_{u}=e^{-r \Delta t}\left(P_{u} f_{u u}+P_{m} f_{u m}+P_{d} f_{u d}\right) \\
f_{m}=e^{-r \Delta t}\left(P_{u} f_{m u}+P_{m} f_{m m}+P_{d} f_{m d}\right) \\
f_{d}=e^{-r \Delta t}\left(P_{u} f_{d u}+P_{m} f_{d m}+P_{d} f_{d d}\right)
\end{gathered}
$$

With these three equation, we get the option price at time zero as follows; 


$$
\begin{aligned}
f=e^{-r \Delta t} & {\left[P_{u} e^{-r \Delta t}\left(P_{u} f_{u u}+P_{m} f_{u m}+P_{d} f_{u d}\right)\right.} \\
& +P_{m} e^{-r \Delta t}\left(P_{u} f_{u m}+P_{m} f_{m m}+P_{d} f_{m d}\right) \\
& \left.+P_{d} e^{-r \Delta t}\left(P_{u} f_{u d}+P_{m} f_{m d}+P_{d} f_{d d}\right)\right] \\
=e^{-2 r \Delta t} & {\left[P_{u}^{2} f_{u u}+P_{m}^{2} f_{m m}+P_{d}^{2} f_{d d}+2 P_{u} P_{m} f_{u m}\right.} \\
& \left.+2 P_{u} P_{d} f_{u d}+2 P_{m} P_{d} f_{m d}\right] .
\end{aligned}
$$

which is a trinomial expansion.

\subsection{Trinomial Expansion}

The equation for a trinomial distribution is given by;

$$
(a+b+c)^{n}=\sum_{h=0}^{n} \sum_{l=0}^{n-h} \frac{n !}{h ! l !(n-h-l) !} a^{h} b^{l} c^{(n-h-l) .}
$$

Therefore our option price $f$ at $t=0$ in general becomes;

$$
\begin{aligned}
f= & e^{-n r \Delta t} \sum_{h=0}^{n} \sum_{l=0}^{n-h}\left(\begin{array}{l}
n \\
h
\end{array}\right)\left(\begin{array}{c}
n-h \\
l
\end{array}\right) \frac{n !}{h ! l !(n-h-l) !} \\
& \times P_{u}^{h} P_{d}^{l} P_{m}^{(n-h-l)} f_{u^{h} d^{l} m^{(n-h-l)}}
\end{aligned}
$$

\subsection{Risk-neutral Probabilities}

In a binomial tree model, the ratios of changes of stock price are $e^{\delta \sqrt{\Delta t}}$ and $e^{-\delta \sqrt{\Delta t}}$. The probabilities of stock price moving up and down are specified so that the expected growth rate of the stock matches the risk-free interest rate.

For trinomial tree model with constant risk-free interest rate and volatility, stock price can go up, remain unchanged or go down by certain ratios. Upward ratio should be greater than $e^{\delta \sqrt{\Delta t}}$ in order to ensure that the risk-neutral probability measure is existing.

Let $P_{u}, P_{m}$ and $P_{d}$ to be the risk-neutral probabilities that the stock price moves up, remain the same or moves down respectively. As an extension of binomial tree model, we approximate the continuous random walk with discrete random walk to be having the same mean and variance. Thus for mean we have;

$$
S_{m} e^{\delta \sqrt{\Delta t}}=E\left(S_{m+1}\right)
$$

where;

$$
S_{m+1}= \begin{cases}S_{m} e^{\beta \delta \sqrt{\Delta t}} & \text { with probability } P_{u} \\ S_{m} & \text { with probility } P_{m} \\ S_{m} e^{-\beta \delta \sqrt{\Delta t}} & \text { with probility } P_{d}\end{cases}
$$

where $\beta>1$, should be there to make risk-neutral probability measure exist.

$$
S_{m} e^{r \sqrt{\Delta t}}=S_{m}\left(P_{u} e^{\beta \delta \sqrt{\Delta t}}+P_{m}+P_{d} e^{-\beta \delta \sqrt{\Delta t}}\right)
$$

implying that;

$$
e^{r \Delta t}=P_{u} e^{\beta \delta \sqrt{\Delta t}}+P_{m}+P_{d} e^{-\beta \delta \sqrt{\Delta t}} .
$$

For variance;

$$
\operatorname{var}\left(S_{(m+1)}\right)=E\left[S_{m+1}\right]^{2}-\left[E\left(S_{m+1}\right)\right]^{2}
$$

where;

$$
S_{m+1}^{2}= \begin{cases}S_{m}^{2} e^{2 \beta \delta \sqrt{\Delta t}} & \text { with probability } P_{1} \\ S_{m}^{2} & \text { with probility } P_{2} \\ S_{m}^{2} e^{-2 \beta \delta \sqrt{\Delta t}} & \text { with probility } P_{3}\end{cases}
$$

Therefore;

$$
\begin{aligned}
\operatorname{var}\left(S_{(m+1)}\right)= & S_{m}^{2}\left[P_{1} e^{2 \beta \delta \sqrt{\Delta t}}+P_{2}+P_{3} e^{-2 \beta \delta \sqrt{\Delta t}}\right] \\
& -S_{m}^{2}\left[P_{1} e^{\beta \delta \sqrt{\Delta t}}+P_{2}+P_{3} e^{-\beta \delta \sqrt{\Delta t}}\right]^{2} .
\end{aligned}
$$

Hence;

$$
\begin{aligned}
\sigma^{2} \Delta t= & {\left[P_{1} e^{2 \beta \delta \sqrt{\Delta t}}+P_{2}+P_{3} e^{-2 \beta \delta \sqrt{\Delta t}}\right] } \\
& -\left[P_{1} e^{\beta \delta \sqrt{\Delta t}}+P_{2}+P_{3} e^{-\beta \delta \sqrt{\Delta t}}\right]^{2} \\
& \Rightarrow\left(P_{1}+P_{3}\right) \beta^{2} \sigma^{2} \Delta t=\sigma^{2} \Delta t .
\end{aligned}
$$

\subsection{Recombining Binomial Trees}

We assume that the volatility is the same in all states in order to make the price ratios for up-steps and down-steps same size irrespective of where they appear in the binomial tree. This means that 2 up-steps and 1 down-step over 3 time periods will take you to the same share price whatever order the steps occur in. Furthermore we assume that the sizes of the up-steps and down-steps are the same in all states.

Therefore we have;

$$
S_{t}=S_{0} u^{N} d^{t-N}
$$

where $N$ is the number of up-steps between time 0 and time $t$. This means we have $n+1$ possible states at time $n$ instead of $2^{n}$. For non-path dependent derivatives we have $C_{n}=f\left(S_{n}\right)$ for some function $f$ : for example, for a European call option we have;

$$
f(x)=\max (x-K, 0)
$$

where $K$ is the strike price.

\subsection{Binomial Distribution}

Under binomial model, the risk-neutral probabilities are equal and all steps are independent of one another. Thus the number of up-steps up to time $t, N$, has binomial distribution with parameters $t$ and $q$.

The price of a derivative at time $t$ is:

$$
\begin{gathered}
V_{t}=e^{-r(n-t)} \sum_{h=0}^{n-t} f\left(S_{t} u^{h} d^{(n-t-h)}\right) \frac{(n-t) !}{h !(n-t-h) !} \\
* q^{h}(1-q)^{n-t-h}
\end{gathered}
$$

\subsection{Trinomial Distribution}

Consider a sequence of $n$ independent trials of an experiment. The binomial distribution arises if each trial 
can result in two outcomes, success or failure, with fixed probability of success $p$ at each trial. If $X$ counts the number of successes, then $X \sim \operatorname{Binomial}(n, p)$.

Now suppose that at each trial there are 3 possibilities, "success", "failure", or "neither" of the two and with the corresponding probabilities $p, q, 1-p-q$ and are the same for all the trials. If we denote "success" by 1 , "failure" by 0 and "neither" by -1 , then the outcome of $n$ trials can be described as a sequence of $n$ numbers, $\omega=\left(i_{1}, i_{2}, \cdots, i_{n}\right)$, where $i_{j}$ takes values $1,0,-1$.

\subsection{Recombining Trinomial Trees}

The importance of trinomial model is majorly limited by the number of states which exist even for relatively small number of time periods up to maturity (i.e $3^{n}$ states). The best solution here is to assume that the volatility is the same in all states and thus the price ratios of up-steps, remaining the same and down-step are the same size, irrespective of where they appear in the trinomial tree. In short, this means that, 1 up-step, 1 same-step and 1 down-step over three time periods will give the same share price whatever order the steps occur.

$$
\begin{aligned}
S_{t} & =S_{0} u^{a} m^{b} d^{(n-a-b)} \\
& =S_{0} u^{a} d^{(n-a-b)}, \text { since } m=1 .
\end{aligned}
$$

Where $a$ is the number of up-steps, $b$ is the number of same-steps. This lead us to having $2 n+1$ possible states at time $n$ and not $3^{n}$. For non-path dependent derivatives we have $C_{n}=f\left(S_{n} u^{a} d^{(n-a-b)}\right)$ for some function $f$. This function together with trinomial distribution give us the price of the derivative.

That is:

$$
\begin{gathered}
V_{t}=e^{-r(n-t)} \sum_{h=0}^{n-t} \sum_{l=0}^{(n-t-h)} f\left(S_{t} u^{h} d^{(n-t-h-l)}\right) \frac{(n-t) !}{h ! l !(n-t-h-l) !} \\
* p^{h} q^{l}(1-p-q)^{n-t-h-l} .
\end{gathered}
$$

which is the price of an option at time $t$. The figure below shows the recombined tree diagram;

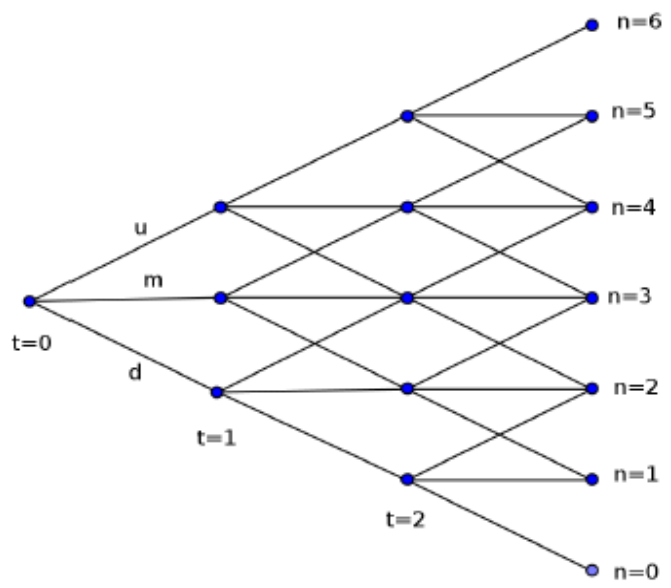

Figure 2. Recombined Tree diagram

\subsection{The Black-Scholes Equation}

The famous Black-Scholes Partial Differential Equation, which is given as

$$
\frac{\partial F}{\partial t}+\frac{1}{2} \sigma^{2} S^{2} \frac{\partial^{2} F}{\partial S^{2}}+r S \frac{\partial F}{\partial S}-r F=0
$$

where $S$ is the asset price, $\sigma$ is the volatility coefficient, $t$ is the time and $r$ is the risk-free interest rate. The equation has an infinite number of solutions. Therefore to obtain a unique solution, hence avoiding the possibilities of arbitrage, boundary conditions must be imposed. These boundary conditions specify how the solution of the problem behaves in some region of the solution domain. We note that the highest derivative with respect to $S$ is a second order derivative and the highest derivative with respect to $t$ is a first order derivative. Hence we impose two conditions about the behaviour of the solution in $S$ and one condition in $t$ as explained in [12].

Let us now introduce the boundary conditions of the PDE as described in [12] for a European put option whose payoff is denoted by $P(S, t)$. The final condition for the option, that is, the value at the terminal point where time $t=T$, is given by

$$
\begin{aligned}
P(S, T) & =(K-S)^{+} \\
& =\max (K-S, 0),
\end{aligned}
$$

where $K$ is the strike price and $S$ is the asset value.

Now suppose that the value of the asset is zero. Then the payoff for the option will automatically be $K$ and the present value of the option received at time $T$ is

$$
P(0, t)=K e^{-r(T-t)} .
$$

Finally, we consider the case when the asset value is large enough. Therefore as $S$ increases, the option value tend to be worthless,

$$
P(S, t)=0 \text { as } S \rightarrow \infty .
$$

The closed form solution for the European put option problem (34) together with the boundary conditions described above is fully explained in [1] where the following assumptions were made in deriving the solution equation.

(i) The risk free interest rate $r$, and asset volatility are constant.

(ii) The underlying asset prices follow a lognormal random walk.

(iii) There are no arbitrage opportunities hence all portfolios would earn equal returns.

(iv) During delta hedging of a portfolio, no transaction costs are incurred.

(v) The short selling of securities is allowed and the assets are divisible.

(vi) No dividends are paid during the entire period of the contract.

(vii) There is continuous trading of the underlying assets.

Therefore the explicit solution for the European put option is given by

$$
P(S, t)=K e^{-r(T-t)} N\left(-d_{2}\right)-S N\left(-d_{1}\right),
$$


where

$$
\begin{aligned}
& d_{1}=\frac{\log (S / K)+\left(r+\frac{1}{2} \sigma^{2}\right)(T-t)}{\sigma \sqrt{T-t}}, \\
& d_{2}=\frac{\log (S / K)+\left(r-\frac{1}{2} \sigma^{2}\right)(T-t)}{\sigma \sqrt{T-t}} .
\end{aligned}
$$

The value of $d_{2}$ can also be approximated as $d_{2} \approx d_{1}-\sigma \sqrt{T-t}$.

The parameter $N(\cdot)$ is the cumulative probability distribution function for a standardized normal random variable

$$
N(x)=\frac{1}{\sqrt{2 \pi}} \int_{-\infty}^{x} e^{-\frac{1}{2} y^{2}} d y .
$$

To obtain the value of $N(x)$ we use the idea described in [13], and we proceed to compute the error function as

$$
\operatorname{erf}(x)=\frac{1}{\sqrt{\pi}} \int_{-x}^{x} e^{-y^{2}} d y,
$$

which simplifies equation (36) to

$$
N(x)=\frac{1}{2}\left(1+\operatorname{erf}\left(\frac{x}{\sqrt{2}}\right)\right) .
$$

\section{Results}

\subsection{Introduction}

In this chapter, we present the results obtained in the calculation of the European put option by using a Trinomial Tree Method. We also segment the time period of an American put option into equal intervals and carry out European put pricing at the end of every interval independently with assumption that every interval is the expiring date of a European put option. We then draw a line graph from these prices of different points. The curve gives us the best prices for an American put option.

The results are then compared to Black - Scholes and Binomial tree method. The numerical solution were obtained by implementing an $\mathrm{R}$ program written for the methods.

\subsection{Trinomial Solution}

In using Trinomial tree method, the results tend to approach explicit solution as we increase the number of time steps. The main steps followed in Trinomial method are;

i. Generate the path for the underlying asset prices by a random walk under a risk neutral world.

ii. Calculate the risk neutral probabilities and take assumption to be all constant throughout.

iii. Evaluate the payoff and get the highest at every node.

iv. Discount the highest payoff at a risk-free interest rate to get the required present value, which is the option price.
Example 1; Trinomial tree method: Consider pricing of a European put option with $S_{0}=100, K=110, r=0.05$, $\sigma=0.25$ and $T=0.5$ yrs. The option price obtained by the Black-Scholes equation is 11.50987 . Therefore using Trinomial method, as the number of time steps increases the results becomes more accurate. The challenge with this is that it takes more computational time when the number of steps is very large.

The table below shows the accuracy with increasing number of time steps of trinomial method. Every step is one week.

Table 1. Trinomial Method

\begin{tabular}{cc}
\hline Number of steps & European Put values \\
\hline 1 & 9.99000 \\
2 & 9.99870 \\
3 & 10.08270 \\
4 & 10.20309 \\
5 & 10.34096 \\
6 & 10.48698 \\
7 & 10.63625 \\
8 & 10.78610 \\
9 & 10.93500 \\
10 & 11.08209 \\
11 & 11.22686 \\
12 & 11.36905 \\
13 & $\mathbf{1 1 . 5 0 8 0 0}$ \\
\hline
\end{tabular}

Using the above results for half a year of the European put price, we come up with the American put price curve (boundary) that give us the max prices at different points.

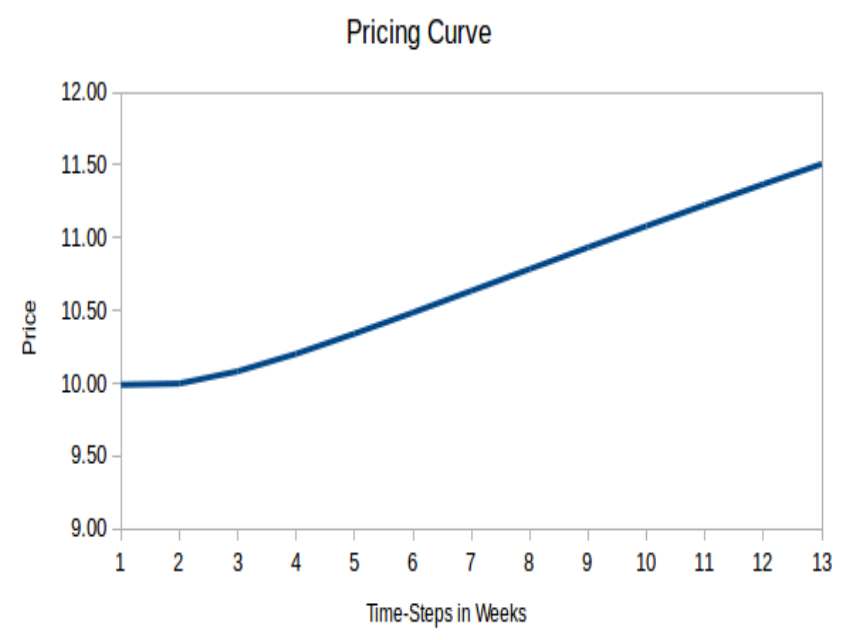

Figure 3. European put option graph

\subsection{Comparison of the Results}

\subsubsection{Example 2; Comparison}

In this example, we make comparison of the European put prices obtained by Black-Scholes formula (35), Binomial tree method and Trinomial tree method. Consider example 1 above, we have parameters $S_{0}=100$, $K=110, \quad r=0.05, \sigma=0.25, T=0.5 y r s$. We will vary the time steps and evaluate the corresponding put prices. The values obtained are shown in the table below. 
Table 2. Comparison of Results

\begin{tabular}{|c|c|c|c|}
\hline Number of steps & Black-Scholes & Trinomial & Binomial \\
\hline 1 & 9.8972 & 9.99000 & 9.894286 \\
\hline 2 & 9.844747 & 9.99870 & 9.788665 \\
\hline 3 & 9.851546 & 10.08270 & 9.806075 \\
\hline 4 & 9.894851 & 10.20309 & 9.894096 \\
\hline 5 & 9.958456 & 10.34096 & 9.918919 \\
\hline 6 & 10.03321 & 10.48698 & 10.05772 \\
\hline 7 & 10.1139 & 10.63625 & 10.09800 \\
\hline 8 & 10.19249 & 10.78610 & 10.23396 \\
\hline 9 & 10.28214 & 10.93500 & 10.25237 \\
\hline 10 & 10.36671 & 11.08209 & 10.40908 \\
\hline 11 & 10.45049 & 11.22686 & 10.42379 \\
\hline 12 & 10.53303 & 11.36905 & 10.57831 \\
\hline 13 & 10.61406 & 11.50800 & 10.58971 \\
\hline 14 & 10.69342 & 11.64529 & 10.74002 \\
\hline 15 & 10.77102 & 11.77919 & 10.7485 \\
\hline 16 & 10.84681 & 11.95048 & 10.89381 \\
\hline 17 & 10.92078 & 12.03902 & 10.89974 \\
\hline 18 & 10.99295 & 12.16502 & 11.03983 \\
\hline 19 & 10.06334 & 12.28851 & 11.04351 \\
\hline 20 & 11.13199 & 12.40959 & 11.17843 \\
\hline 21 & 11.19894 & 12.52832 & 11.18013 \\
\hline 22 & 11.26423 & 12.71021 & 11.31006 \\
\hline 23 & 11.32793 & 12.84360 & 11.30998 \\
\hline 24 & 11.39006 & 11.39006 & 11.43517 \\
\hline 25 & 11.4507 & 13.21441 & 11.4335 \\
\hline 26 & 11.50987 & 13.48689 & 11.55419 \\
\hline
\end{tabular}

\section{Recommendation}

We recommend the use of American price curve (boundary) to find a fair price for American put options. The asset price returns are assumed to follow a normal distribution. In reality, the returns are known to follow one of the heavy tailed distributions. Therefore we recommend the models above be modified to incorporate the concept of the distribution of asset returns.

\section{Conclusion}

The pricing of derivatives has been made easier by the development of Black-Scholes model. The implementation of the Trinomial and Binomial tree methods made it easier to make a comparison of the results obtained by these numerical methods to the explicit solution obtained by using the Black-Scholes formula. We observed that the results obtained were approximately equal to the explicit solution. The Trinomial method converges to the answer two times faster than the Binomial and the Black-Scholes methods. This paper presented a recombining trinomial tree for European options valuation with unchanging volatilities. This was done by letting the time steps regular and risk neutral probabilities remaining the same for the entire contract time. This recombining trinomial tree is more flexible and easy to use than just a tree with a lot of nodes which are more the same and thus suitable for predicting prices of options.

\section{Acknowledgments}

We would like to thank God for everything He has done for us up to this point. We also pass our regards to all those who have made worthy contribution to this work. A lot of thanks goes to our colleagues who have been so supportive and provided guidance whenever needed. Special thanks go to our parents and family for their support and encouragement throughout the preparation of this paper.

\section{References}

[1] P Wilmott, JN Dewynne, and SD Howison. Option pric- ing, mathematical methods and computation, 1993.

[2] Fischer Black, Michael C Jensen, Myron Scholes, et al. The capital asset pricing model: Some empirical tests. Studies in the theory of capital markets, 81(3):79-121, 1972.

[3] Robert C Merton et al. Theory of rational option pricing. Theory of Valuation, pages 229-288, 1973

[4] John C Cox, Stephen A Ross, and Mark Rubinstein. Op- tion pricing: A simplified approach. Journal of financial Economics, 7(3): 229-263, 1979.

[5] Phelim P Boyle. Options: A monte carlo approach. Jour- nal of financial economics, 4(3): 323-338, 1977.

[6] Phelim P Boyle and Sok Hoon Lau. Bumping up against the barrier with the binomial method. The Journal of Derivatives, 1(4): 6-14, 1994.

[7] Hu Xiaoping, Guo Jiafeng, Du Tao, Cui Lihua, and Cao Jie. Pricing options based on trinomial markov tree. Dis- crete Dynamics in Nature and Society, 2014, 2014.

[8] F AitSahlia and Tze L Lai. Valuation of discrete barrier and hindsight options. Journal of Financial Engineering, 6(2): 169177, 1997.

[9] Harish S Bhat and Nitesh Kumar. Option pricing under a normal mixture distribution derived from the markov tree model. European Journal of Operational Research, 223(3):762-774, 2012

[10] XIONG Bing-zhong. A trinomial option pricing model based on bayesian markov chain monte carlo method. Journal of Jiaxing University, (6):9, 2012.

[11] Fei Lung Yuen and Hailiang Yang. Pricing asian options and equity-indexed annuities with regime switching by the trinomial tree method. North American Actuarial Journal, 14(2): 256-272, 2010.

[12] Paul Wilmott. The mathematics of financial derivatives: a student introduction. Cambridge University Press, 1995.

[13] George Marsaglia et al. Evaluating the normal distribu- tion. Journal of Statistical Software, 11(4):1-7, 2004. 